\title{
Comparison of Rat Lung Tissue Following Inhalation and Intratracheal Administration of Metal Oxide Nanoparticles
}

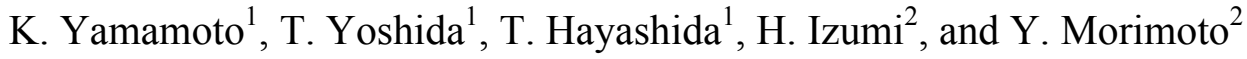 \\ 1. National Institute of Advanced Industrial Science and Technology (AIST), Tsukuba, Japan \\ 2. University of Occupational and Environmental Health, Kitakyushu, Japan
}

Industrial applications of nanoparticles were reported in many fields recently. However, the effects of nanoparticles on the human body are not fully understood. The gold standard test to estimate the harmful effect of respirable chemicals is inhalation studies, which can provide reliable and informative data about the effects on the human body because of their similarity to human exposure. However it is difficult to examine the hazard and risk evaluation of lots of nanoparticles using inhalation studies because of expensive cost, and necessity of specific apparatus and experts. On the other hand intratracheal administration studies, of which chemicals was inserted into trachea or pharyngeal cavity, have been used for dose-dependency of pulmonary toxicity and the mechanism of lung disorder due to exposure of chemicals with known quantity. In this study, in order to examine whether or not intratracheal administration studies can be useful for determining the harmful effect nanoparticles, we performed inhalation and intratracheal administration studies using the same nanoparticle samples and examined the similarities and differences of lung tissue between both studies.

The $\mathrm{NiO}$ and $\mathrm{TiO}_{2}$ nanoparticles were examined in this study. The $\mathrm{NiO}$ or $\mathrm{TiO}_{2}$ suspension of 0.2 $\mathrm{mg} / 0.4 \mathrm{ml}$ was intratracheally instilled once to F344 rats (12 weeks old). The negative control groups received distilled water. The $\mathrm{NiO}$ and $\mathrm{TiO}_{2}$ were aggregated in water suspension. The size of $\mathrm{NiO}$ aggregate in suspension is $40-50 \mathrm{~nm}$, and that of $\mathrm{TiO}_{2}$ aggregate is $20-80 \mathrm{~nm}$. The aerosols of $\mathrm{NiO}$ and $\mathrm{TiO}_{2}$ nanoparticles were generated from their water suspension. The main diameter of NiO aerosol was $20 \mathrm{~nm}$, and that of $\mathrm{TiO}_{2}$ aerosol was $30 \mathrm{~nm}$. Rats were exposed to the aerosol for 6 hours a day, 5 days a week, for 4 weeks in a whole-body exposure chamber. The average mass concentrations of aerosol were $1.65 \mathrm{mg} / \mathrm{m}^{3}$ for $\mathrm{NiO}$, and $1.84 \mathrm{mg} / \mathrm{m}^{3}$ for $\mathrm{TiO}_{2}$. Animals were dissected at 3 days, 1 month, 3 months later. Lung tissues after the tests were observed by TEM. The lung tissues were fixed using a perfusion system using a 4\% paraformaldehyde solution, and were then post-fixed using a $1 \%$ osmium tetroxide solution. They were subsequently dehydrated in ethanol, followed by embedding in epoxy resin. Ultrathin sections were cut with a diamond knife using microtomy. Thin sectioning specimens were stained with a $2 \%$ uranyl acetate solution and a mixed solution of $0.3 \%$ lead nitrate and $0.3 \%$ lead acetate, all at room temperature. Conventional TEM observation was performed with H-7600 (Hitachi, Japan) at an accelerating voltage of 80kV.

Lung tissue TEM images of $\mathrm{NiO}$ intratracheal administration group 1 month after exposure were shown in Figure 1 (a)-(b). Figure 1 (b) was magnified image of boxed area in Figure 1 (a). Alveolar macrophages with black particles in phagolysosomes were observed, which were indicated with arrows in Figure 1 (b). These black particles are not observed in the negative control group, which were instilled pure water. According to X-ray analysis in TEM, it is clarified that these black particles are $\mathrm{NiO}$. Uptake of $\mathrm{NiO}$ by alveolar macrophages is also observed in the high dose group. Lung tissue TEM images of $\mathrm{NiO}$ inhalation group 1 month after exposure were shown in Figure 1 (c)-(d). Figure 1 (d) was magnified image of boxed area in Figure 1 (c). Uptake of NiO particles in alveolar macrophages is also observed. Mount of $\mathrm{NiO}$ particles in each alveolar macrophage in inhalation study is less than that in intratracheal administration study. We think this is because $\mathrm{NiO}$ is localized in lung of intratracheal administration study, and alveolar macrophage in intratracheal 
administration takes up more $\mathrm{NiO}$ particles compared with inhalation study. In both intratracheal administration and inhalation study, the same uptake of $\mathrm{NiO}$ by alveolar macrophages is observed. In cases of intratracheal administration and inhalation of $\mathrm{TiO}_{2}, \mathrm{TiO}_{2}$ particles are observed in phagolysosomes of alveolar macrophages, and uptake of $\mathrm{TiO}_{2}$ by alveolar macrophages is observed in both studies. More mount of $\mathrm{TiO}_{2}$ particles are observed in an alveolar macrophage in intratracheal administration because of the same reason in $\mathrm{NiO}$ study.

In summary, we performed inhalation and intratracheal administration studies using the same samples of $\mathrm{NiO}$ and $\mathrm{TiO}_{2}$ as chemicals with high and low toxicities, respectively. Both of $\mathrm{NiO}$ and $\mathrm{TiO}_{2}$ nanoparticles are taken up by alveolar macrophages, and shown as same behavior in inhalation and intratracheal administration studies [1].

\section{References:}

[1] This work is supported by "Development of innovative methodology for safety assessment of industrial nanomaterials" by Ministry of Economy, Trade and Industry (METI) of Japan.

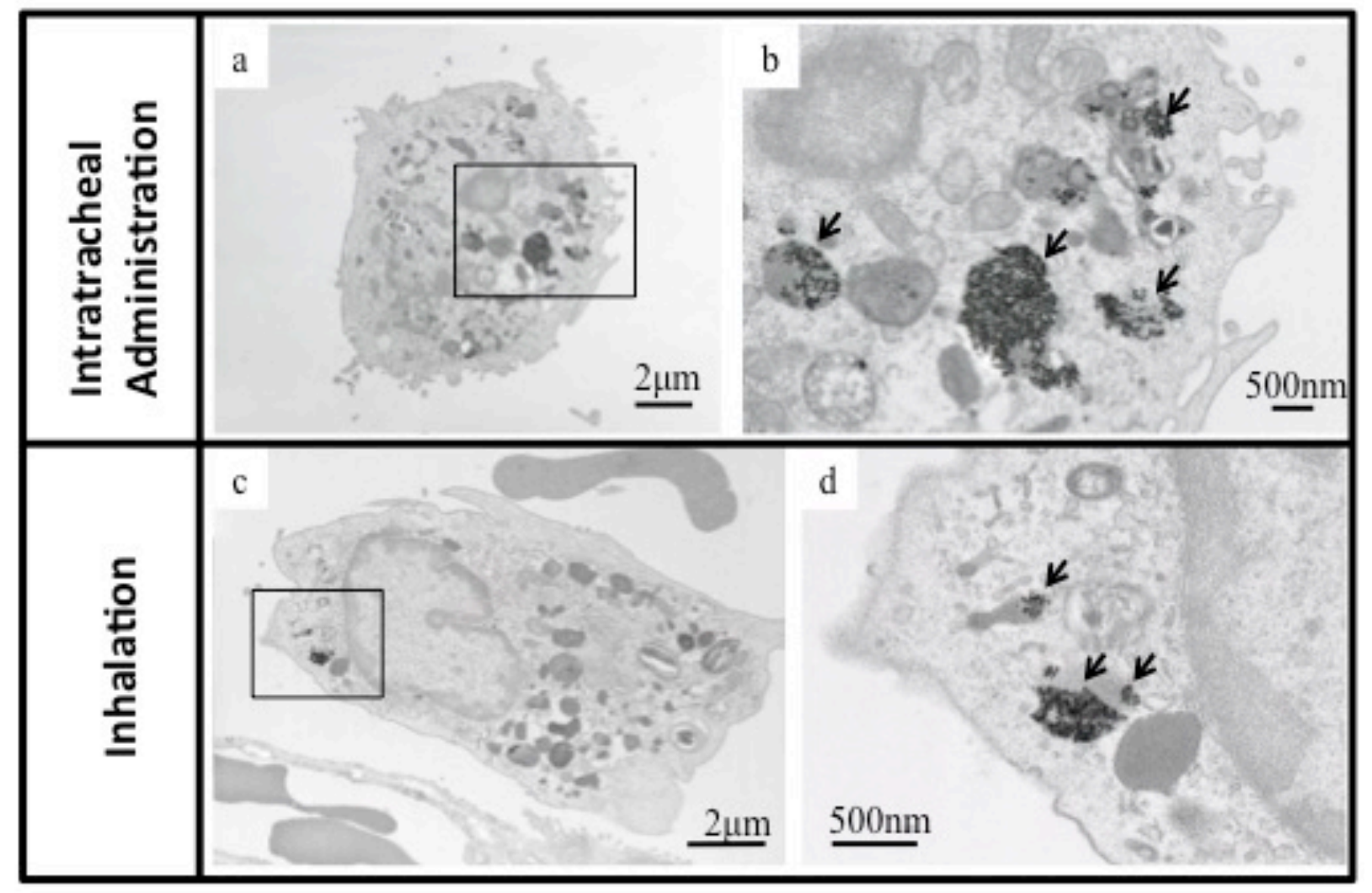

Figure 1. Lung tissue TEM image (a), and magnified image of boxed area in Fig. (a) (b) of 1 month after $\mathrm{NiO}$ intratracheal administration. Lung tissue TEM image (c), and magnification image of boxed area in Fig. (c) (d) of 1 month after NiO inhalation. 